\title{
Positive periodic solution of higher-order functional difference equation
}

\author{
Mei-Lan Tang and Xin-Ge Liu*
}

\author{
* Correspondence: \\ liuxgliuhua@163.com \\ School of Mathematical Science \\ and Computing Technology, \\ Central South University Changsha, \\ Hunan 410083, China
}

\begin{abstract}
Based on a fixed point theorem in a cone, a new sufficient condition for the existence of a positive periodic solution to a class of higher-order functional difference equations is established in this article. The result obtained in this article is different from the existing results in previous literature.

Mathematic Subject Classification 2000: 34k13; MSC 39A70.
\end{abstract}

Keywords: positive periodic solution, fixed point theorem, cone, existence

\section{Introduction}

The existence of positive periodic solutions of discrete mathematical models such as the discrete model of blood cell production and the single-species discrete periodic population model has been studied extensively in recent years (see [1-8], for example). Most of these discrete mathematical models are first-order functional difference equations. Relatively, few articles focused on the existence of positive periodic solutions of higher-order functional difference equations. In 2010, Wang and Chen [9] have studied the existence of positive periodic solutions for the following general higher-order functional difference equation

$$
x(n+m+k)-a x(n+m)-b x(n+k)+a b x(n)=f(n, x(n-\tau(n)))
$$

where $a \neq 1, b \neq 1$ are positive constants, $\tau: Z \rightarrow Z$ and $\tau(n+\omega)=\tau(n), f(n+\omega, u)=$ $f(n, u)$ for any $u \in R, \omega, m, k \in N$ where $N$ denotes the set of positive integers. Based on fixed point theorem in a cone $[10,11]$, some new sufficient conditions on the existence of positive periodic solutions to the higher-order functional difference equation (1) are obtained. However, the main results in [9] require that $a$ should be positive constant, $l$ should satisfy condition $l=\omega$ where $l=\frac{\omega}{(m, \omega)}$ and $(m, \omega)$ are the greatest common divisor of $m$ and $\omega$. In fact, in most cases, $m$ and $\omega$ do not satisfy such severe constraint $l=\omega$. In general, $l \leq \omega$. In this article, we consider the following higherorder functional difference equation

$$
x(n+m+k)-a(n+m) x(n+m)-b x(n+k)+a(n) b x(n)=f(n, x(n-\tau(n)))
$$


where $b \neq 1$ is positive constant, $a: Z \rightarrow R_{+}$with $a(n) \neq 1$ and $a(n+\omega)=a(n), \tau$ : $Z \rightarrow Z$ and $\tau(n+\omega)=\tau(n), f(n+\omega, u)=f(n, u)$ for any $u \in R, k, \omega, m \in N$ where $N$ denotes the set of positive integers.

The purpose of this article is to consider the existence of positive periodic solution of higher-order functional difference equation (2), we will remove the constrains on $a$ and $l$ in [9]. We will replace constant $a$ in [9] with function $a(n)$. At same time, we will remove the unreasonable assumption $l=w$. Based on a fixed point theorem in a cone, a new sufficient condition is established for the existence of positive periodic solutions for higher-order functional difference equation.

\section{Some preparation}

Let $X$ be the set of all real $\omega$ periodic sequences, then $X$ is a Banach space with the maximum norm $\|x\|=\max _{n \in[0, \omega-1]}|x(n)|$.

Lemma 1 (Deimling [10]) Let $X$ be a Banach space and $K$ be a cone in X. Suppose $\Omega_{1}$ and $\Omega_{2}$ are open subsets of $X$ such that $0 \in \Omega_{1} \subset \bar{\Omega}_{1} \subset \Omega_{2}$ and suppose that

$$
\Phi: K \cap\left(\bar{\Omega}_{2} \backslash \Omega_{1}\right) \rightarrow K
$$

is a completely continuous operator such that

(i) $\|\Phi u\| \leq\|u\|$ for $u \in K \cap \partial \Omega_{1}$ and there exists $\psi \in K \backslash\{0\}$ such that $x \neq \Phi x+\lambda \psi$ for $x \in K \cap \partial \Omega_{2}$ and $\lambda>0$; or

(ii) $\|\Phi u\| \leq\|u\|$ for $u \in K \cap \partial \Omega_{2}$ and there exists $\psi \in K \backslash\{0\}$ such that $x \neq \Phi x+\lambda \psi$ for $x \in K \cap \partial \Omega_{1}$ and $\lambda>0$.

Then, $\Phi$ has a fixed point in $K \cap\left(\bar{\Omega}_{2} \backslash \Omega_{1}\right)$.

Let $d \in N$. Consider the equation

$$
x(n+d)=c x(n)+\gamma(n)
$$

where $\gamma \in X$. Set $(d, \omega)$ as the greatest common divisor of $d$ and $\omega, p=\omega /(d, \omega)$.

Lemma 2 [9]Assume that $0<c \neq 1$, then (3) has a unique periodic solution

$$
x(n)=\left[c^{-p}-1\right]^{-1} \sum_{i=1}^{p} c^{-i} \gamma(n+(i-1) d) .
$$

Let $y(n)=x(n+k)-a(n) x(n), \bar{a}=\max _{1 \leq n \leq \omega} a(n), \underline{a}=\min _{1 \leq n \leq \omega} a(n)$, then (2) can be rewritten as

$$
\left\{\begin{array}{l}
x(n+k)=\underline{a} x(n)+y(n)+[a(n)-\underline{a}] x(n), \\
y(n+m)=b y(n)+f(n, x(n-\tau(n))) .
\end{array}\right.
$$


Let $h=\frac{\omega}{(k, \omega)}, l=\frac{\omega}{(m, \omega)}$. Assume that $x \in X$ solution of (2), then $y \in X$. From Lemma 2, we have

$$
\begin{aligned}
& x(n)=\left[\underline{a}^{-h}-1\right]^{-1} \sum_{i=1}^{h} \underline{a}^{-1}\{y(n+(i-1) k)+[a(n+(i-1) k)-\underline{a}] x(n+(i-1) k)\}, \\
& y(n)=\left[b^{-l}-1\right]^{-1} \sum_{i=1}^{l} b^{-i} f(n+(i-1) m, x(n+(i-1) m-\tau(n+(i-1) m))) .
\end{aligned}
$$

If $f(n, x(n-\tau(n))) \geq 0$ and $0<b<1$, then $y(n) \geq 0$.

We introduce the following conditions:

(H) $0<a(n)<1,0<b<1, h=\omega$ and $f: R \times(0,+\infty) \rightarrow[0,+\infty)$ is continuous.

Define the operator $T$ by

$$
\begin{aligned}
(T x)(n)= & \frac{\underline{a}^{h} b^{l}}{\left(1-\underline{a}^{h}\right)\left(1-b^{l}\right)} \sum_{i=1}^{h} \underline{a}^{-i} \sum_{j=1}^{l} b^{-j} f(n+(i-1) k+(j-1) m, \\
& x(n+(i-1) k+(j-1) m-\tau(n+(i-1) k+(j-1) m))) \\
& +\frac{\underline{a}^{h}}{\left(1-\underline{a}^{h}\right)} \sum_{i=1}^{h} \underline{a}^{-i}[a(n+(i-1) k)-\underline{a}] x(n+(i-1) k) .
\end{aligned}
$$

Define the cone by

$$
K=\{x \in X, x(n) \geq \delta\|x\|\}
$$

where $\delta=\underline{a}^{h} b^{l}\left(1-\underline{a}^{h}\right)\left(1-b^{l}\right) / \omega$.

Lemma 3 Assume that $(H)$ holds and $0<r_{1}<r_{2}$, then $T: \bar{K}_{r_{2}} \backslash K_{r_{1}} \rightarrow$ Kis completely continuous, where $K_{r}=\{x \in K:\|x\|<r\}$ and $\bar{K}_{r}=\{x \in K:\|x\| \leq r\}$.

Proof Since $0<a(n)<1$, then $0<\underline{a}<1$. Noting that $0<b<1$ and $f(n, x(n-\tau(n))) \geq$ 0 , we have $y(n) \geq 0$. So $(T x)(n) \geq 0$ on $[0, \omega-1]$. Since $\tau(n+\omega)=\tau(n)$ and $f(n+\omega, u)$ $=f(n, u)$ for any $u>0,(T x)(n+\omega)=(T x)(n)$ for $x \in X$. Since $l=\frac{\omega}{(m, \omega)} \leq \omega$ we have

$$
\sum_{j=1}^{l} f(n+(j-1) m, x(n+(j-1) m-\tau(n+(j-1) m))) \leq \sum_{j=1}^{\omega} f(j, x(j-\tau(j))) .
$$

On the other hand, from $(\mathrm{H}), h=\frac{\omega}{(k, \omega)}=\omega$, we have

$$
\sum_{i=1}^{h} f(n+(i-1) k, x(n+(i-1) k-\tau(n+(i-1) k)))=\sum_{i=1}^{\omega} f(i, x(i-\tau(i)))
$$


and

$$
\sum_{i=1}^{h}[a(n+(i-1) k)-\underline{a}] x(n+(i-1) k)=\sum_{i=1}^{\omega}[a(i)-\underline{a}] x(i) .
$$

For any $x \in \bar{K}_{r_{2}} \backslash K_{r_{1}}$,

$$
\begin{aligned}
& (T x)(n)=\frac{\underline{a}^{h} b^{l}}{\left(1-\underline{a}^{h}\right)\left(1-b^{l}\right)} \sum_{i=1}^{h} \underline{a}^{-i} \sum_{j=1}^{l} b^{-j} f(n+(i-1) k+(j-1) m, \\
& x(n+(i-1) k+(j-1) m-\tau(n+(i-1) k+(j-1) m))) \\
& +\frac{\underline{a}^{h}}{\left(1-\underline{a}^{h}\right)} \sum_{i=1}^{h} \underline{a}^{-i}[a(n+(i-1) k)-\underline{a}] x(n+(i-1) k) \\
& \leq \frac{\underline{a}^{h} b^{l}}{\left(1-\underline{a}^{h}\right)\left(1-b^{l}\right)} \underline{a}^{-h} b^{-l} \sum_{i=1}^{h} \sum_{j=1}^{l}\{f(n+(i-1) k+(j-1) m, \\
& x(n+(i-1) k+(j-1) m-\tau(n+(i-1) k+(j-1) m)))\} \\
& +\frac{\underline{a}^{h}}{\left(1-\underline{a}^{h}\right)} \underline{a}^{-h} \sum_{i=1}^{h}[a(n+(i-1) k)-\underline{a}] x(n+(i-1) k) \\
& =\frac{\underline{a}^{h} b^{l}}{\left(1-\underline{a}^{h}\right)\left(1-b^{l}\right)} \underline{a}^{-h} b^{-l} \sum_{j=1}^{l} \sum_{i=1}^{h}\{f(n+(i-1) k+(j-1) m, \\
& x(n+(i-1) k+(j-1) m-\tau(n+(i-1) k+(j-1) m)))\} \\
& +\frac{\underline{a}^{h}}{\left(1-\underline{a}^{h}\right)} \underline{a}^{-h} \sum_{i=1}^{\omega}(a(i)-\underline{a}) x(i) \\
& \leq \frac{1}{\left(1-\underline{a}^{h}\right)\left(1-b^{l}\right)} \sum_{j=1}^{\omega} \sum_{i=1}^{\omega} f(i, x(i-\tau(i))) \\
& +\frac{1}{\left(1-\underline{a}^{h}\right)} \sum_{i=1}^{\omega}(a(i)-\underline{a}) x(i) \\
& \leq \frac{\omega}{\left(1-\underline{a}^{h}\right)\left(1-b^{l}\right)} \sum_{i=1}^{\omega} f(i, x(i, \tau(i))) \\
& +\frac{1}{(1-\underline{a} h)} \sum_{i=1}^{\omega}(a(i)-\underline{a}) x(i) \\
& \leq \frac{\omega}{\left(1-\underline{a}^{h}\right)\left(1-b^{l}\right)} \sum_{i=1}^{\omega}\{f(i, x(i, \tau(i)))+(a(i)-\underline{a}) x(i)\} \text {. }
\end{aligned}
$$

So

$$
\|T x\| \leq \frac{\omega}{\left(1-\underline{a}^{h}\right)\left(1-b^{l}\right)} \sum_{i=1}^{\omega}\{f(i, x(i-\tau(i)))+(a(i)-\underline{a}) x(i)\} .
$$


At the same time

$$
\begin{aligned}
& (T x)(n) \geq \frac{\underline{a}^{h} b^{l}}{\left(1-\underline{a}^{h}\right)\left(1-b^{l}\right)} \underline{a}^{-1} b^{-1} \sum_{i=1}^{h} \sum_{j=1}^{l}\{f(n+(i-1) k+(j-1) m, \\
& x(n+(i-1) k+(j-1) m-\tau(n+(i-1) k+(j-1) m)))\} \\
& +\frac{\underline{a}^{h}}{\left(1-\underline{a}^{h}\right)} a^{-1} \sum_{i=1}^{h}[a(n+(i-1) k)-\underline{a}] x(n+(i-1) k) \\
& =\frac{\underline{a}^{h} b^{l}}{\left(1-\underline{a}^{h}\right)\left(1-b^{l}\right)} \underline{a}^{-1} b^{-1} \sum_{j=1}^{l} \sum_{i=1}^{h}\{f(n+(i-1) k+(j-1) m, \\
& x(n+(i-1) k+(j-1) m-\tau(n+(i-1) k+(j-1) m)))\} \\
& +\frac{\underline{a}^{h}}{\left(1-\underline{a}^{h}\right)} \underline{a}^{-1} \sum_{i=1}^{\omega}(a(i)-\underline{a}) x(i) \\
& \geq \frac{\underline{a}^{h}}{\left(1-\underline{a}^{h}\right)} \frac{l b^{l}}{\left(1-b^{l}\right)} \sum_{i=1}^{\omega} f(i, x(i-\tau(i))) \\
& +\frac{\underline{a}^{h}}{\left(1-\underline{a}^{h}\right)} \sum_{i=1}^{\omega}(a(i)-\underline{a}) x(i) \\
& \geq \frac{\underline{a}^{h}}{\left(1-\underline{a}^{h}\right)} \sum_{i=1}^{\omega}\left[\frac{b^{l}}{\left(1-b^{l}\right)} f(i, x(i, \tau(i)))+(a(i)-\underline{a}) x(i)\right] \\
& \geq \underline{a}^{h} \sum_{i=1}^{\omega}\left[b^{l} f(i, x(i-\tau(i)))+(a(i)-\underline{a}) x(i)\right] \\
& \geq \underline{a}^{h} b^{l} \sum_{i=1}^{\omega}[f(i, x(i-\tau(i)))+(a(i)-\underline{a}) x(i)] .
\end{aligned}
$$

We have

$$
(T x)(n) \geq \delta\|T x\| .
$$

Thus $T: \bar{K}_{r_{2}} \backslash K_{r_{1}} \rightarrow K$ is well defined. Since $X$ is finite-dimensional Banach space, one can easily show that $T$ is completely continuous. This completes the proof.

We can easily obtain the following result.

Lemma 4 The fixed point of $T$ in $K$ is a positive periodic solution of (2).

\section{Main result}

Let

$$
\begin{aligned}
& \varphi(s)=\max \{f(n, u), n \in[0, \omega-1], u \in[\delta s, s]\} \\
& \psi(s)=\min \left\{\frac{f(n, u)}{u}, n \in[0, \omega-1], u \in[\delta s, s]\right\}
\end{aligned}
$$

Let $\bar{a}=\max _{1 \leq n \leq \omega} a(n), \underline{a}=\min _{1 \leq n \leq \omega} a(n)$.

Theorem 1 Assume that $(H)$ holds and there exist two positive constants $\alpha, \beta$ with $\alpha$ $\neq \beta$ such that 


$$
\varphi(\alpha) \leq(\bar{a}-1)(b-1) \alpha, \psi(\beta) \geq(\underline{a}-1)(b-1)
$$

Then (2) has at least one positive $\omega$-periodic solution $x$ with $\min \{\alpha, \beta\} \leq\|x\| \leq \max$ $\{\alpha, \beta\}$.

Proof Without loss of generality, we assume that (H) holds, $\alpha<\beta$. Obviously, $0<\bar{a}<1,0<\underline{a}<1$. We claim that:

(i) $\|T x\| \leq\|x\|, x \in \partial K_{\alpha}$,

(ii) $x \neq T x+\lambda \cdot 1, \forall x \in \partial K_{\beta}, 1 \in K$ and $\lambda>0$.

From (7), we have that

$$
\begin{array}{ll}
f(n, x) \leq(\bar{a}-1)(b-1) \alpha, \quad \forall 0 \leq n \leq \omega-1, \quad \forall \delta \alpha \leq x \leq \alpha, \\
f(n, x) \geq(\underline{a}-1)(b-1) x, \quad \forall 0 \leq n \leq \omega-1, \quad \forall \delta \beta \leq x \leq \beta .
\end{array}
$$

In order to prove (i), let $x \in \partial K_{\alpha}$, then $\|x\|=\alpha$ and $\delta \alpha \leq x(n) \leq \alpha$ for $0 \leq n \leq \omega-1$. So

$$
\begin{aligned}
(T x)(n)= & \frac{\underline{a}^{h} b^{l}}{\left(1-\underline{a}^{h}\right)\left(1-b^{l}\right)} \sum_{i=1}^{h} \underline{a}^{-i} \sum_{j=1}^{l} b^{-j} f(n+(i-1) k+(j-1) m, \\
& x(n+(i-1) k+(j-1) m-\tau(n+(i-1) k+(j-1) m))) \\
& +\frac{\underline{a}^{h}}{\left(1-\underline{a}^{h}\right)} \sum_{i=1}^{h} \underline{a}^{-i}[a(n+(i-1) k)-\underline{a}] x(n+(i-1) k) \\
\leq & \frac{\underline{a}^{h} b^{l}}{\left(1-\underline{a}^{h}\right)\left(1-b^{l}\right)} \sum_{i=1}^{h} \underline{a}^{-i} \sum_{j=1}^{l} b^{-j}\{(\bar{a}-1)(b-1) \alpha\} \\
& +\frac{\underline{a}^{h}}{\left(1-\underline{a}^{h}\right.} \sum_{i=1}^{h} \underline{a}^{-i}[\bar{a}-\underline{a}]\|x\| \\
\leq & \left\{\frac{b^{l}}{\left(1-b^{l}\right)}(1-b) \sum_{j=1}^{l} b^{-j}\right\} \frac{\underline{a}^{h}}{\left(1-\underline{a}^{h}\right)} \sum_{i=1}^{h} \underline{a}^{-i}\{(1-\bar{a}) \alpha\} \\
& +\frac{\underline{a}^{h}}{\left(1-\underline{a}^{h}\right)} \sum_{i=1}^{h} \underline{a}^{-i}[\bar{a}-\underline{a}] \alpha \\
= & \frac{\underline{a}^{h}}{\left(1-\underline{a}^{h}\right)} \sum_{i=1}^{h} \underline{a}^{-i}[1-\underline{a}] \alpha \\
= & \alpha .
\end{aligned}
$$

It follows that

$$
\|T x\| \leq\|x\|, x \in \partial K_{\alpha} .
$$

Next, let $\psi=1 \in K$ in Lemma 1 , we prove (ii). If not, there exists $u_{\mathrm{o}} \in \partial K_{\beta}$ and $\lambda_{\mathrm{o}}>$ 0 such that

$$
u_{0}=\left(T u_{0}\right)(n)+\lambda_{0} .
$$

Since $u_{\mathrm{o}} \in \partial K_{\beta}$, then $\left\|u_{\mathrm{o}}\right\|=\beta$ and $\delta \beta \leq u_{\mathrm{o}}(n) \leq \beta$. Put $u_{\mathrm{o}}(n)=\min \left\{u_{\mathrm{o}}(i) \mid 0 \leq i \leq \omega-\right.$ $1\}$ for some $n \in[0, \omega-1]$. Noting that $u_{\mathrm{o}}(n)>0$ and $0<\underline{a}<1$, we have 


$$
\begin{aligned}
u_{0}(n)= & \left(T u_{0}\right)(n)+\lambda_{0} \\
= & \frac{\underline{a}^{h} b^{l}}{\left(1-\underline{a}^{h}\right)\left(1-b^{l}\right)} \sum_{i=1}^{h} \underline{a}^{-i} \sum_{j=1}^{l} b^{-j} f(n+(i-1) k+(j-1) m, \\
& \left.u_{0}(n+(i-1) k+(j-1) m-\tau(n+(i-1) k+(j-1) m))\right) \\
& +\frac{\underline{a}^{h}}{\left(1-\underline{a}^{h}\right)} \sum_{i=1}^{h} \underline{a}^{-i}[a(n+(i-1) k)-\underline{a}] u_{0}(n+(i-1) k)+\lambda_{0} \\
\geq & \frac{\underline{a}^{h} b^{l}}{\left(1-\underline{a}^{h}\right)\left(1-b^{l}\right)} \sum_{i=1}^{h} \underline{a}^{-i} \sum_{j=1}^{l} b^{-j}\{f(n+(i-1) k+(j-1) m, \\
& \left.\left.u_{0}(n+(i-1) k+(j-1) m-\tau(n+(i-1) k+(j-1) m))\right)\right\}+\lambda_{0} \\
\geq & \frac{\underline{a}^{h} b^{l}}{\left(1-\underline{a}^{h}\right)\left(1-b^{l}\right)} \sum_{i=1}^{h} \underline{a}^{-i} \sum_{j=1}^{l} b^{-j}(\underline{a}-1)(b-1) u_{0}(n+(i-1) k \\
& +(j-1) m-\tau(n+(i-1) k+(j-1) m))+\lambda_{0} \\
\geq & u_{0}(n)+\lambda_{0}
\end{aligned}
$$

which implies that $u_{\mathrm{o}}(n)>u_{\mathrm{o}}(n)$, a contradiction.

Therefore, by Lemma $1, T$ has a fixed point $x \in K_{\beta} \backslash K_{\alpha}$. Furthermore, $\alpha \leq\|x\| \leq \beta$ and $x(n) \geq \delta \alpha$, which means that $x$ is one positive periodic solution of (2). The proof is completed.

\section{Example}

Now, an example is given to demonstrate our result.

Example 1 Consider the difference equation

$$
x(n+m+k)-a(n+m) x(n+m)-b x(n+k)+a(n) b x(n)=f(n, x(n-\tau(n)))
$$

where $b=1 / 2, m=3, k=5, \omega=6, \tau: Z \rightarrow Z$ and $\tau(n+\omega)=\tau(n), a: Z \rightarrow R_{+}$with $a(n)=\frac{1}{2}+\frac{1}{16} \cos \frac{n \pi}{3}, f(n, u)=\left(1-\frac{7}{16}\right)\left(1-\frac{1}{2}\right) u^{3}\left[1+\frac{1}{2}(-1)^{n} \cos \frac{\pi u}{3}\right]$.

Obviously, $a(n+\omega)=a(n+6)=a(n), f(n+\omega, u)=f(n+6, u)=f(n, u)$ for any $u \in$

$R$.

$$
h=\frac{\omega}{(k, \omega)}=\frac{6}{(5,6)}=6, l=\frac{\omega}{m, \omega}=\frac{6}{(3,6)}=2 . \bar{a}=\max _{1 \leq n \leq \omega} a(n)=\frac{9}{16}, \underline{a}=
$$

$$
\min _{1 \leq n \leq \omega} a(n)=\frac{7}{16}, \delta=\left(\frac{7}{16}\right)^{6}\left(\frac{1}{2}\right)^{2}\left[1-\left(\frac{7}{16}\right)^{6}\right]\left[1-\left(\frac{1}{2}\right)^{2}\right] / 6
$$

Let $\alpha=\frac{1}{2}$, then

$$
\begin{aligned}
\varphi(\alpha) & =\varphi\left(\frac{1}{2}\right) \\
& \leq\left(1-\frac{7}{16}\right)\left(1-\frac{1}{2}\right)\left(\frac{1}{2}\right)^{3}\left[1+\frac{1}{2}\right] \\
& =\left(1-\frac{7}{16}\right)\left(1-\frac{1}{2}\right)\left(\frac{1}{2}\right)^{2} \frac{3}{4} \\
& <\left(\frac{9}{16} \frac{1}{2}\right)\left(1-\frac{1}{2}\right) \frac{1}{2} \\
& <\left(1-\frac{9}{16}\right)\left(1-\frac{1}{2}\right) \frac{1}{2} .
\end{aligned}
$$

So $\varphi(\alpha) \leq(\bar{a}-1)(b-1) \alpha$. 
Let $\beta=\frac{2}{\delta}$. If $u \in[\delta \beta, \beta]$, then $u \geq 2$. Furthermore,

$$
\begin{aligned}
\psi(\beta) & \geq\left(1-\frac{7}{16}\right)\left(1-\frac{1}{2}\right)\left(\frac{2^{3}}{2}\right)\left[1-\frac{1}{2}\right] \\
& =2\left(1-\frac{7}{16}\right)\left(1-\frac{1}{2}\right) \\
& >\left(1-\frac{7}{16}\right)\left(1-\frac{1}{2}\right) .
\end{aligned}
$$

So $\psi(\beta) \geq(\underline{a}-1)(b-1)$.

By Theorem 1 in this article, (12) has at least one positive 6-periodic solution.

\section{Acknowledgements}

The authors would like to thank the reviewers for their valuable comments and constructive suggestions. This study was partly supported by the ZNDXQYYJJH under grant no. 2010QZZD015, Hunan Scientific Plan under grant no. 2011FJ6037, NSFC under grant no. 61070190 and NFSS under grant no. 10BJL020.

\section{Authors' contributions}

All authors contributed equally to the manuscript and read and approved the final draft.

\section{Competing interests}

The authors declare that they have no competing interests.

Received: 19 May 2011 Accepted: 21 November 2011 Published: 21 November 2011

\section{References}

1. Cheng, SS, Zhang, G: Positive periodic solutions of a discrete population model. Funct Diff Equ. 7(34), 223-230 (2000)

2. Pielou, EC: Mathematics Ecology. Wiley-Interscience, New York (1997)

3. Li, Y, Zhu, L: Existence of positive periodic solutions for difference equations with feedback control. Appl Math Lett. 18(1), 61-67 (2005). doi:10.1016/j.aml.2004.09.002

4. Saito, Y, Ma, W, Hara, T: Necessary and sufficient condition for permanence of a Lotka-Volterra discrete system with delays. J Math Anal Appl. 256, 162-174 (2001). doi:10.1006/jmaa.2000.7303

5. Zhang, R, Wang, Z, Chen, Y, Wu, J: Periodic solution of a single species discrete population model with periodic harvest stock. Comput Math Appl. 39, 77-90 (2000)

6. Meng, X, Chen, L: Periodic solution and almost periodic solution for a nonautonomous Lotka-Volterra dispersal system with infinite delay. J Math Anal Appl. 339, 125-145 (2008). doi:10.1016/j.jmaa.2007.05.084

7. Raffoul, YN: Positive periodic solutions of nonlinear functional difference equations. Electron J Diff Equ. 55, 1-8 (2002)

8. Jiang, D, O'Regan, D, Agarwal, RP: Optimal existence theory for single and multiple positive periodic solutions to functional difference equations. Appl Math Comput. 161, 441-462 (2005). doi:10.1016/j.amc.2003.12.097

9. Wang, W, Chen, X: Positive periodic solutions for higher order functional difference equation. Appl Math Lett. 23 1468-1472 (2010). doi:10.1016/j.aml.2010.08.013

10. Deimling, K: Functional Analysis. Springer, Berlin (1985)

11. Guo, D, Lakshmikantham, V: Nonlinear Problem in Abstract Cones. Academic Press, New York (1988)

Cite this article as: Tang and Liu: Positive periodic solution of higher-order functional difference equation. Advances in Difference Equations 2011 2011:56.

\section{Submit your manuscript to a SpringerOpen ${ }^{\odot}$ journal and benefit from:}

- Convenient online submission

- Rigorous peer review

- Immediate publication on acceptance

- Open access: articles freely available online

- High visibility within the field

- Retaining the copyright to your article

Submit your next manuscript at $\boldsymbol{s p r i n g e r o p e n . c o m ~}$ 